\title{
Electro interstitial scan system: assessment of 10 years of research and development
}

This article was published in the following Dove Press journal:

Medical Devices: Evidence and Research

2 March 2012

Number of times this article has been viewed

\section{Albert Maarek \\ Research and Development, LD Technology, Miami, FL, USA}

Correspondence: Albert Maarek New World Tower, 100 N. Biscayne Blvd, Suite 500, Miami, FL 33।32, USA

Tel +l 3053799900

Fax + I 3054683956

Email albert.ldteck@gmail.com
Background: Ten years of research and development have allowed an understanding of how the electro interstitial scan (EIS) works and what its clinical applications may be.

Materials and methods: The EIS is a galvanic skin response device. The measurements are performed by electrical stimulation of the post sympathetic cholinergic fiber with weak DC current and voltage $1.28 \mathrm{~V}$ applied during 2 minutes and in bipolar mode.

Current scientific knowledge: EIS electrical measurements are related to: (1) the concentration of free chloride ions in the interstitial fluid, which affects the transfer of electrical current and the ratio intensity/voltage; (2) the morphology of the interstitial fluid, which is related to the electrical dispersion calculated from the Cole equation ( $\alpha$ parameter); (3) electrical stimulation, which causes a change in sweat rate at the passive electrodes - post sympathetic cholinergic fiber electrical stimulation appears to be responsible for activating M2 receptors, which regulate nitric oxide (NO) production in the endothelial cell and cause vasodilation and a released sweat response; and (4) the electrochemical redox reactions (electrolysis) of the released sweat on electrodes, which are different on the bulk of the metal electrodes $\left(\mathrm{O} 2+\left[4 \mathrm{H}^{+}\right]+\left[4 \mathrm{e}^{-}\right]\right)$ and on the $\mathrm{Ag} / \mathrm{AgCl}$ disposable electrodes ( $\mathrm{AgCl}$ precipitation).

Results: For each of the EIS clinical results, various explanations were posited, such as: (1) electrical stimulation of the postsympathetic cholinergic fiber-activating NO production in the endothelial cell, which causes vasodilation and a released sweat response (diabetes detection); (2) estimation of interstitial fluid's acid-base balance, which is reflected in an electrochemical reaction on the bulk of the electrodes through the released sweat (prostate cancer detection); (3) estimation of cerebral interstitial fluid chloride ions (detection of ADHD in children); and (4) estimation of the morphology of the interstitial fluid (selective serotonin reuptake inhibitor treatment response).

Conclusion: After 10 years of development, the analysis of current scientific knowledge and results of clinical investigations have allowed a better understanding of EIS electrical measurements.

Keywords: EIS, electro interstitial scan, electrochemical redox reactions, postsympathetic cholinergic electrical stimulation, ADHD, SSRI treatment responses, prostate cancer, diabetes

\section{Introduction}

Galvanic skin response (GRS) is a method of measuring the electrical conductance of the skin related to the sweat gland responses. ${ }^{1}$ The sweat glands are controlled by the post sympathetic cholinergic system. Thus, skin conductance has been used as an indication of psychological or physiological arousal. The first device used to measure the electrical conductance between two electrodes, was essentially a type of ohmmeter. 
The scientific study of GSR began in the early 1900s. Reich studied GSR at the Psychological Institute at the University of Oslo in 1935, and confirmed the existence of a bio-electrical charge behind his concept of vegetative pleasurable streaming. ${ }^{2}$ GSR was used for a variety of research in the 1960s and 1970s, with a decline in use as more sophisticated techniques (such as EEG and MRI) replaced it in many areas of psychological research.

The EIS is classified as GSR, but unlike the ohmmeter, it was designed using a scientific basis including the electrochemistry, bioimpedance, and physiology of the sweat glands, with the objective being to produce reproducible measurements of conductance for use as markers of disease or indicators of treatment response. Also, EIS data are able to be sent to a computer for signal processing, analysis, and follow-up.

Ten years of development and clinical investigations have led to an understanding of how EIS works and what its clinical applications may be. In this paper, we present the results of this work including interpretation of conductance measurements and a discussion of clinical investigations.

\section{Materials and methods}

The EIS (Electro Interstitial Scan, LD Technology, Miami, FL) system includes a USB "plug and play" hardware device, an interface box, and six tactile electrodes and cables. The six electrodes are placed on the skin with two on the forehead (one on the left, one on the right), two in contact with the palms of the hands, and two in contact with the soles of the feet. The electrodes on the hands and feet are at least $250 \mathrm{~cm}^{2}$ and are made of stainless steel. The forehead electrodes are disposable (single use), are $15.75 \mathrm{~cm}^{2}$, and are made of $\mathrm{Ag} / \mathrm{AgCl}$. Software is installed on the computer, and the USB port provides data transfer as well as power to the hardware.

\section{Contraindications}

- Dermatological lesions in contact with the electrodes

- Excessive perspiration (hyperhidrosis)

- The patient using a cardiac pacemaker, being connected to electronic life support devices or any implanted electronic device, or the presence of defibrillators

- Patients who are unable to sit

- Metal pins or prostheses on the extremities or joints

- Pregnancy

- The absence of one or more limbs.

\section{Description of the test procedure}

1. The device and accessories are cleaned/disinfected and then dried in the air. This must be performed before each patient.

2. The examination area should be comfortable, free of portable electric heaters, and not drafty.

3. The measurement is carried out with the patient in a seated position.

4. The patient is barefoot and removes all metal objects (wristwatch, bracelets, rings, etc) which may come into contact with the electrodes.

5. Any creams, makeup, or foundation on the forehead are removed by wiping with alcohol, and then letting the area air dry.

6. The feet and hands are cleaned with alcohol, and then air-dried.

7. The patient must register and provide details including date of birth, weight, and height.

\section{Measurement process}

Automatic testing before measurement indicates that the hardware and software are operating correctly, and that the connection to the patient is acceptable. Once the EIS begins, a sequence of successive measurements is performed on eleven pathways of the body using weak DC current $(200 \mu \mathrm{A})$ and an imposed voltage of $1.28 \mathrm{~V}$ between the six tactile electrodes (Table 1). The software changes the polarity for each pathway, first from anode to cathode and then from cathode to anode. The conductance of each pathway is measured every 32 milliseconds for 1 second for each polarity.

\section{Description of the measurement process}

The DC current circuit is shown in Figure 1 (steps of the current transfer from active electrode to passive electrode).

\section{Steps I and 2: entrance of the current from the active electrode}

Because voltage lower than $10 \mathrm{~V}$ cannot penetrate the stratum corneum, ${ }^{3}$ the only way for the current to enter the body is through the eccrine sweat ducts. This route represents the physiological pathway of the interstitial fluid, ie, the source of the eccrine sweat. ${ }^{4}$

\section{Step 3: pathway into the human body between the two electrodes}

1. According to Fricke's circuit, the plasma membrane acts as an insulator and a DC current is not able to penetrate the 
Table I Sequence of measurement of the II pathways

\begin{tabular}{lll}
\hline Pathways & & \\
\hline Anode & Direction & Cathode \\
\hline I. Left forehead & $\leftrightarrow$ & 2. Left hand \\
3. Right forehead & $\leftrightarrow$ & 4. Right hand \\
5. Left hand & $\leftrightarrow$ & 6. Left foot \\
7. Right hand & $\leftrightarrow$ & 8. Right foot \\
9. Left forehead & $\leftrightarrow$ & 10. Right forehead \\
II. Left hand & $\leftrightarrow$ & 12. Right hand \\
13. Left foot & $\leftrightarrow$ & 14. Right foot \\
15. Right hand & $\leftrightarrow$ & 16. Left forehead \\
17. Left hand & $\leftrightarrow$ & 18. Right forehead \\
19. Right foot & $\leftrightarrow$ & 20. Left hand \\
21. Left foot & $\leftrightarrow$ & 22. Right hand \\
\hline
\end{tabular}

cell, so most of the current flows around the cell through the interstitial fluid. ${ }^{5,6}$

2. In the interstitial fluid, free ions are able to conduct a current in the presence of an external electrical field. We can consider biological tissue to be an ionic conductor, both electrically and macroscopically. The total ionic conductance of a solution depends on the concentration, activity, charge, and mobility of all free ions in the solution..$^{5,6}$

3. The ionic concentration is proportional to the ratio intensity/voltage, so that as the ionic concentration increases, more intensity and less voltage are observed. 5,6

4. Electrical dispersion: The cell membrane has the ability to store capacitive energy (that is, it has dielectric or insulator properties). The cell membrane is the cellular structure that makes the largest contribution to the dielectric behavior of living tissue. Living tissue is considered to be a dispersive medium..$^{7-9}$

In 1940, Cole introduced the first mathematical expression able to describe the "depressed semicircles" found experimentally. This is known as the Cole equation. ${ }^{7-9}$

$$
\mathrm{Z}=R_{\infty}+\frac{\Delta R}{1+(j \omega \tau)^{\alpha}}, \quad \Delta R=R_{0}-R_{\infty}
$$

where $\mathrm{Z}$ is the impedance value at frequency $\omega, j$ is the complex number $(-1) 1 / 2, R_{\infty}$ is the impedance at infinite frequency, $R_{0}$ is the impedance at zero frequency, $\tau$ is the characteristic time constant, and $\alpha$ is a dimensionless parameter with a value between 0 and 1 . The $\alpha$ value can also be regarded as a parameter denoting the derivation from the Fricke-Morse model. That is, the Cole equation with $\alpha=1$ is equal to the Fricke-Morse model. ${ }^{10}$

In the case of living tissues, the spectral width of the electrical bioimpedance dispersions (closely related to the $\alpha$ parameter in the Cole equation) evolves during the ischemic periods. Simulations indicate that the dispersion width could be determined by the morphology of extracellular spaces. ${ }^{11}$

\section{Steps 4, 5, and 6: Process of the exit of the current to the passive electrode}

Electrical stimulation causes a released sweat response response. ${ }^{12}$ Mechanical shear stress causes a phosphorylation cascade that removes phosphate groups from proteins and

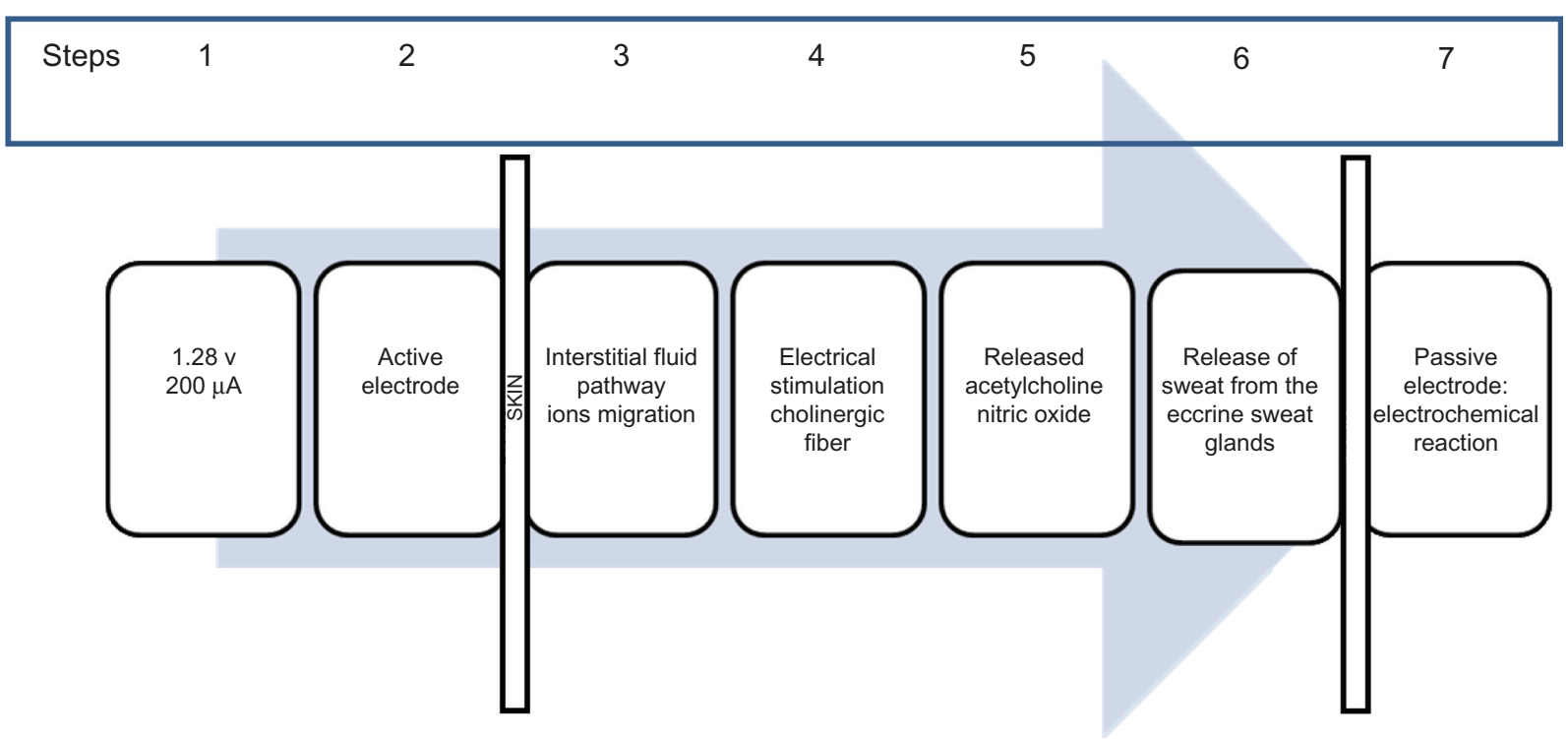

Figure I Steps of the current transfer from anode to cathode and then from cathode to anode for each pathway. 
kinases, activating endothelial NO synthase. NO is produced, facilitating the release of cyclic guanosine monophosphate, and a change in potassium permeability. The relaxation of the smooth muscle and vasodilatation of the vessels allows an exchange between vessels and the sweat glands, which facilitates the production of sweat. ${ }^{12,13}$

The release of acetylcholine (Ach) is regulated by the hypothalamus and, in the case of the sweat response, acts as a response to an increase in blood and/or skin temperature. ${ }^{13}$ The mechanical response initiated by shear stress is not dependent on a temperature increase, and in the GSR method, it appears that electrical stimulation acted as a mechanical shear stress activator. ${ }^{13}$

Step 7: Electrochemical reactions on the bulk of the passive electrode (electrolysis)

Analysis of the DC current in the cathode and anode in electrolytic solution $\mathrm{Na}^{+} \mathrm{Cl}^{-}$using metal electrodes: the electrochemical window is defined by both reduction and oxidation of water according to the following reactions. ${ }^{14}$

\section{At the cathode}

$\mathrm{Na}^{+}$ions are not discharged at the cathode as sodium is not very electropositive, which means that it takes a lot of energy and a large negative voltage on the cathode to impose electrons on $\mathrm{Na}^{+}$ions. At a lower voltage, dissolved oxygen is reduced and water molecules are decomposed. Both processes are linked with non-charged species that are transported to the electron transfer sites by diffusion rather than by migration.

When the voltage supply is adjusted $(>1 \mathrm{~V})$, oxygen reduction is performed. $\mathrm{Na}^{+}$ions are necessary for the conductance of the solution.

The water electrochemical reaction (reduction) at the cathode is $2 \mathrm{H}_{2} \mathrm{O}+\left(2 \mathrm{e}^{-}\right)=\left(\mathrm{H}_{2}\right)+\left(2 \mathrm{OH}^{-}\right)$.

\section{At the anode}

The current at the anode is due to the discharge of $\mathrm{Cl}^{-}$ions. Chloride is highly electropositive, and less energy is necessary for taking electrons from the chloride ions than from water molecules. The water electrochemical reaction (oxidation) at the anode is $2 \mathrm{H}_{2} \mathrm{O}=\left(\mathrm{O}_{2}\right)+\left(4 \mathrm{H}^{+}\right)+\left(4 \mathrm{e}^{-}\right)$.

In the interstitial fluid, $\mathrm{Na}^{+}$represents $96 \%$ of the positive free ions, and $\mathrm{Cl}^{-}$and $\mathrm{HCO}_{3}^{-}$ions represent $96 \%$ of the negative free ions. ${ }^{14}$

The interstitial fluid can be considered an electrolytic solution of $\mathrm{Na}^{+}$and $\mathrm{Cl}^{-}$because $\mathrm{HCO}_{3}^{-}$and $\mathrm{Na}^{+}$ions may not be discharged and they do not contribute very much to the DC current transfer. ${ }^{3}$ The in vitro electrochemical model described above can be applied only for the $\mathrm{Cl}^{-}$ions at the anode. ${ }^{14}$

\section{Analysis of the DC current in anode in electrolytic solution $\mathrm{Na}^{+} \mathrm{Cl}^{-}$using $\mathrm{Ag} / \mathrm{AgCl}$}

The Cotlove coulometric chloride titrator method measures the total chloride concentration. ${ }^{15}$ With this method, the passage of a constant direct current between $\mathrm{Ag} / \mathrm{AgCl}$ electrodes produces silver ions. The free silver ions at the anode react with the chloride forming silver chloride as follows:

$$
\mathrm{Ag} \rightarrow \mathrm{Ag}^{+} \mathrm{Ag}^{+}+\left(\mathrm{Cl}^{-}\right) \rightarrow \mathrm{AgCl}
$$

After all the chloride has combined with $\mathrm{Ag}^{+}$, free silver ions accumulate and precipitate, causing an increase in current across the electrodes and indicating the end point of the reaction. The technique is already used in vitro to assess the $\mathrm{Cl}^{-}$concentration in small quantities of sweat.

\section{Results \\ Cycle of measurements}

The full cycle comprises 22 conductance measurements of the 11 pathways measured in the polarity anode-cathode in 1 second, and in the polarity cathode-anode (also in 1 second). The measurements' values are displayed in a scale from 0 to 100 for each pathway and a fast Fourier transform graph at three frequencies (Figure 2).

\section{Signal processing analysis}

The following results were analyzed for each segment/ pathway:

- Conductance in $\mu \mathrm{S}$ of each anode-cathode pathway (SDC+)

- Conductance in $\mu \mathrm{S}$ of each cathode-anode pathway (SDC-)

- Numeric value in CU (conventional unit) (delta SDC+/ $\mathrm{SDC}-$ )

- Dispersion of each pathway in CU (calculated using the Cole equation) ( $\alpha$ parameter)

\section{Frequency or spectral analysis} of the entire cycle of measurements

\section{Application of the fast Fourier transform}

(FFT) to the entire signal

The FFT includes EIS HF (high frequencies from 0.1875 to $0.50 \mathrm{~Hz}$ ), EIS LF (low frequencies from 0.05 to $0.1875 \mathrm{~Hz}$ ), and EIS VLF (very low frequencies from 0 to $0.05 \mathrm{~Hz}$ ). 


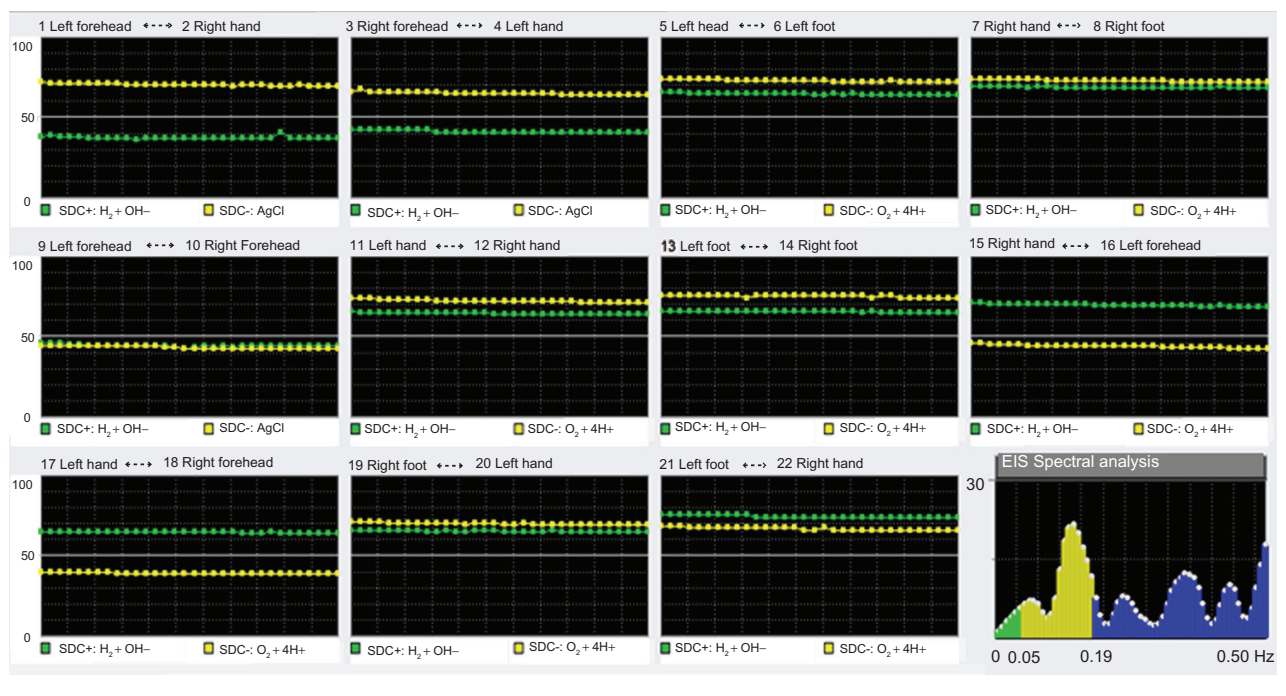

Figure 2 Results of a cycle of measurements in numeric values and fast Fourier transform graph at three frequencies. Abbreviations: EIS, electro interstitial scan; SDC, standard deviation of conductance from anode to cathode.

\section{EIS reproducibility}

Electrical stimulation caused an almost linear increase of sweat output after stimulation. Thereafter, sweat output decreased exponentially to baseline levels within 2 minutes. Repetitive electrical stimulation at 2.75-minute intervals reliably evoked a sweat response. No significant difference in sweat output was obtained between the three stimulations performed within each session.

\section{EIS clinical investigations}

Statistical analysis has been performed in 600 healthy subjects to determine the normal range of EIS conductance for each pathway. The results of EIS research have been published in peer-reviewed journals and show interesting clinical applications.

\section{Improvement of the total PSA value in screening of prostate cancer}

This research ${ }^{16}$ showed that the PSA test had a sensitivity of $73.9 \%$ and specificity of $51.9 \%$ using a cutoff value of 0.4 and a sensitivity of $52.2 \%$ and specificity of $81.5 \%$ using a cutoff value of $5.7(P=0.03)$. The delta of the electrical conductance (DE) of the left foot-right foot pathway had a sensitivity of $62.5 \%$ and specificity of $85.2 \%$, with a cutoff value of $-5(P=0.0001)$. Algorithms comprising the delta of electrical conductance and PSA showed a sensitivity of $91.5 \%$ and a specificity of $59.3 \%$, with a cutoff value of $-10.52(P=0.0003)$.

\section{Measurable markers of the response of SSRI treatment ${ }^{17}$}

Fifty-nine subjects (mean age 47 years, range 17-76 years; 38 women) diagnosed with major depression disorder by psychiatric assessment at the Botkin Hospital according to DSM-IV and CGI (Clinical Global Impression) were recorded with the EIS System before undergoing antidepressant SSRI treatment.

SSRI treatment follow-up was undertaken with both the EIS bioimpedance measurements (electrical conductance and dispersion $\alpha$ parameter) and by treatment responses based on the Hamilton Depression Scale (Ham-D) and CGI every 15 days over 60 days.

At day 45, two groups were established: Group 1: group with treatment response and Group 2: group with nontreatment response. At day 60, another two groups were established: Group 3: group with treatment response and Group 4: group with non-treatment response

Comparing Group 1 and Group 2, electrical conductance measurement of the pathway between the two forehead electrodes had a specificity of $72 \%$ and a sensitivity of $85.3 \%$ $(P=0.0001)$, with a cutoff of 4.32 . Comparing Group 3 and Group 4, electrical conductance measurements in the same pathway had a specificity of $47.6 \%$ and a sensitivity of $76.3 \%$ $(P=0.16)$, with a cutoff of 5.92. Comparing Group 1 and Group 2 , the electrical dispersion $\alpha$ parameter of the pathway between the two disposable forehead electrodes had a specificity of $80 \%$ and a sensitivity of $85.2 \%(P=0.0001)$ with a cutoff of 0.678 . Comparing Group 3 and Group 4, the electrical dispersion $\alpha$ parameter of the same pathway had a specificity of $100 \%$, a sensitivity of $89.5 \%(P=0.0001)$, and a cutoff of 0.692 .

\section{Identifying attention deficit/hyperactivity disorder (ADHD) in children ${ }^{18}$}

Comparing ADHD group and control group children, the mean of the conductance measurements of two pathways 
between the forehead electrodes (from left forehead to right forehead and from right forehead to left forehead) in the ADHD group was 33.11 micro Siemens $(\mu \mathrm{S})$ (range 2-113 $\mu \mathrm{S})$. This was significantly higher $(P=0.001)$ than the mean of the conductance measurements of two pathways between the forehead electrodes of the control group $(2.75 \mu \mathrm{S}$, range $1.75-27.4 \mu \mathrm{S})$. In terms of the receiver operator characteristic (ROC) results, comparing the two groups using the reference of the mean of conductance measurements of the two pathways between the forehead electrodes, the test showed a specificity of $98 \%$ and sensitivity of $80 \%(P=0.0001)$ with a cutoff value at $7.4 \mu \mathrm{S}$.

\section{Predicting the sympathetic system activity level in healthy subjects ${ }^{19}$}

The correlation between EIS HF spectrum analysis and HRV LF spectrum analysis variable was $r=0.76(P<0.001)$. Utilizing EIS HF spectrum analysis as the independent variable and the HRV LF as the dependent variable in a linear regression, the model was statistically significant $(\mathrm{F}[1,49]=63.8$, $P=0.001)$. The adjusted $R^{2}$ value was 0.56 . Finally, a $t$-test indicated that EIS HF spectrum analysis was a significant predictor of HRV LF spectrum analysis $(t=8.0, P=0.001)$.

\section{Equivalent galvanic skin response has been used in diabetes screening ${ }^{20}$}

The experiment showed that electrical stimulation did cause an increase in sweat response between the two electrodes. Control subjects showed a $20.2 \%$ increase in sweat rate and people with diabetes showed an $18.2 \%$ increase in sweat rate.

\section{Discussion}

The EIS has been shown to have the capacity to predict the activity level of the sympathetic nervous system. ${ }^{20}$ This GRS device's capacity for this has been reported in numerous studies, ${ }^{21,22}$ and can be explained by the hypothalamus response to the temperature change according to the sympathetic system activity, via the cholinergic fiber and sweat released.

EIS conductance is a measurable marker of ADHD in children. ${ }^{19}$ The EIS conductance measurements between the two frontal electrodes are increased in children with ADHD, and this could be due to increased cerebral interstitial $\mathrm{Cl}^{-}$ ions measured by the Cotlove coulometric chloride titrator method in the released sweat, and therefore by decrease of intracellular $\mathrm{Cl}^{-}$ions. It has been shown that the cerebral intracellular $\mathrm{Cl}^{-}$ions are correlated to cerebral oxygen/ glucose. ${ }^{23}$ In children with ADHD, the concentration of interstitial $\mathrm{Cl}^{-}$ions is increased and cerebral oxygen/ glucose is also increased, which increases ATPase pump activity and neuronal excitability, and decreases cerebral dopamine levels. ${ }^{24}$

EIS conductance also increases after 45 days of SSRI treatment. ${ }^{18}$ Research shows that the increased intracellular $\mathrm{Cl}^{-}$ions decreased cerebral oxygen/glucose and neural excitability. ${ }^{24}$ Therefore the decreased interstitial $\mathrm{Cl}^{-}$ions measured by the Cotlove coulometric chloride titrator method in the released sweat in the depression study ${ }^{18}$ are related to decreased cerebral oxygen/glucose and neural excitability, which increases GABA, which can provoke depression. The marker $\alpha$ parameter between the two frontal electrodes is more sensitive and specific than the conductance measurement in the response of SSRI treatment after 45 and 60 days of treatment. ${ }^{18}$

The dispersion $\alpha$ parameter may increase in the forehead pathway during SSRI treatment, which may be due to changes in the morphology of the extracellular spaces between the two electrodes. ${ }^{11}$ A decrease in cerebral serotonin could be related to interstitial fluid volume and cerebral neural plasticity. ${ }^{25}$

The delta of conductance in the foot pathway was the marker used in screening for prostate cancer. According to the electrochemical redox reaction at the bulk of the anode (production $\mathrm{OH}^{-}$) and cathode (production $\mathrm{H}^{+}$) electrodes, the markers could be related to acid production in the foot pathway. ${ }^{26}$ As well as this, the galvanic skin response technique provides markers to screen for diabetes. ${ }^{21}$ Research has shown a decrease in conductance measurements between the foot electrodes in type 2 diabetes patients. ${ }^{27}$

The reported decreases in conductance measurements in the foot pathway could be related to decreases in $\mathrm{NO},{ }^{27}$ which means that patients with diabetes have lower rates of sweating, ${ }^{28}$ and with moderate fiber damage, the sweat response may be lost. ${ }^{28}$ The poor glycemic control seen in diabetes causes precapillary damage, which in turn leads to microvascular damage. ${ }^{28}$ Precapillary damage inhibits the normal functioning of endothelial cells and blocks the normal NO pathways that cause vasodilatation. Endothelial cell damage influences vascular tone by causing a loss of distensibility in the blood vessels, which affects the ability of the vessels to dilate. ${ }^{28}$

In summary, the electrical measurements discussed in this paper can detect five physiological indicators of disease. The results of clinical investigations may be due to a number of causes which are set out in Figure 3. 


\begin{tabular}{|c|c|c|c|c|c|}
\hline $\begin{array}{l}\text { Electrical } \\
\text { measurements }\end{array}$ & $\begin{array}{l}\text { Interstitial fluid } \\
\mathrm{Cl}^{-} \text {ions } \\
\text { concentration } \\
\mathrm{Ag} / \mathrm{AgCl} \\
\text { electrodes }\end{array}$ & $\begin{array}{l}\text { Interstitial fluid } \\
\text { morphology } \\
\mathrm{Ag} / \mathrm{AgCl} \\
\text { electrodes }\end{array}$ & $\begin{array}{l}\text { Nitric oxide } \\
\text { released } \\
\text { Stainless steel } \\
\text { electrodes }\end{array}$ & $\begin{array}{l}\text { Sweat rate } \\
\text { response } \\
\text { Stainless steel } \\
\text { electrodes }\end{array}$ & $\begin{array}{l}\text { Sweat acid/base } \\
\text { balance } \\
\text { Stainless steel } \\
\text { electrodes }\end{array}$ \\
\hline & 4 & 4 & 4 & 4 & $\boldsymbol{\uparrow}$ \\
\hline $\begin{array}{l}\text { Clinical } \\
\text { investigation } \\
\text { results }\end{array}$ & $\begin{array}{l}\text { ADHD children } \\
P=0.0001\end{array}$ & $\begin{array}{l}\text { SSRIs treatment } \\
\text { response } \\
P=0.0001\end{array}$ & $\begin{array}{l}\text { Diabetes } \\
P=0.01\end{array}$ & $\begin{array}{l}\text { Sympathetic system level } \\
\text { activity } P=0.001 \\
\text { (healthy subjects) }\end{array}$ & $\begin{array}{l}\text { Prostate cancer } \\
P=0.001\end{array}$ \\
\hline
\end{tabular}

Figure 3 Relationship between the electrical measurements and the clinical investigations results. Abbreviations: ADHD, attention deficit/hyperactivity disorder; SSRIs, selective serotonin reuptake inhibitors.

\section{Conclusion}

After 10 years of development, EIS electrical measurements are better understood due to the results of clinical investigations. EIS electrical measurements have interesting clinical applications in different medical fields such as use as markers of ADHD in children and the treatment responses of SSRIs, or estimating the activity of the sympathetic system and screening for prostate cancer. To improve the specificity and sensitivity of the screening tests in diabetes, the EIS data were analyzed with data of other technologies such as heart rate variability analysis, bioimpedance to assess the body composition, and photoelectrical plethysmography.

\section{Acknowledgments}

Thanks are extended to all the people who contributed to the EIS clinical investigations.

\section{Disclosure}

The author reports no conflict of interest in any EIS clinical investigation. The manuscript is only an assessment and analysis between the current scientific knowledge related to bio-electrical measurement and clinical investigation results.

\section{References}

1. Martini FH, Bartholomew EF. Essentials of Anatomy and Physiology. 4th edition. San Francisco, CA: Benjamin Cummings, Pearson Education; 2007.

2. Reich W. Experimental investigation of the electrical functions of sexuality and anxiety. J Orgonomy. 1969;3:132-154.

3. Chizmadzhev YA, Indenbom AV, Kuzmin PI, Galichenko SV, Weaver JC, Potts RO. Electrical properties of skin at moderate voltages: contribution of appendageal macropores. Biophys J. 1998;74(2): 843-856.

4. Hashimoto K. Demonstration of the intercellular spaces of the human eccrine sweat gland by lanthanum. I. The secretory coil. J Ultrastruct Res. 1971;36:249-262.

5. Gabriel S, Lau RW, Gabriel C. The dielectric properties of biological tissues: III. Parametric models for the dielectric spectrum of tissues. Phys Medicine Biol. 1996;41:2271-2293.
6. Foster KR, Schwan HP. Dielectric properties of tissues and biological materials: a critical review. Crit Rev Biomed Eng. 1989;17(1): 25-104.

7. Cole KS. Electric phase angle of cell membranes. J Gen Physiol. 1932;15(6):641-649.

8. Cole KS, Li CL, Bak AF. Electrical analogues for tissues. Exp Neurol. 1969;24(3):459-473.

9. Cole KS, Cole RH. Dispersion and absorption in dielectrics. I. Alternating current characteristics. J Chem Phys. 1941;9:341-351.

10. Soucy KG, Ryoo S, Benjo A, et al. Impaired shear stress-induced nitric oxide production through decreased NOS phosphorylation contributes to age-related vascular stiffness. J Appl Physiol. 2006;101(6):1751-1759.

11. Ivorra A, Genesca M, Sola A, et al. Bioimpedance dispersion width as a parameter to monitor living tissues. Physiol Meas. 2005; 26:1-9.

12. Donadio V, Lenzi P, Montagna P, Falzone F, Baruzzi A, Liguori R. Habituation of sympathetic sudomotor and vasomotor skin responses: neural and non-neural components in healthy subjects. Clin Neurophysiol. 2005;116(11):2542-2549.

13. Petrofsky J, Hinds CM, Batt J, Prowse M, Suh HJ. The interrelationships between electrical stimulation, the environment surrounding the vascular endothelial cells of the skin, and the role of nitric oxide in mediating the blood flow response to electrical stimulation. Med Sci Monit. 2007;13(9):CR391-CR397.

14. Grimmes S, Martinsen ØG. Electrolytics. In: Bioimpedance and Bioelectricity Basics. Maryland Heights, MO: Academic Press; 2008.

15. Cotlove E, Nishi HH. Automatic titration with direct read-out of chloride concentration. Clin Chem. 1961;7:285-291.

16. Abreu DS. Bioimpedance and chronoamperometry as an adjunct to prostate-specific antigen screening for prostate cancer. Cancer Manag Res. 2011;3:109-116.

17. Aleexev VG, Kuznecova LV. Bioimpedance in monitoring of effects of selective serotonin reuptake inhibitor treatment. Psychol Res Behav Manag. 2011;4:81-86.

18. Caudal F. New marker using bioimpedance technology in screening for attention deficit/hyperactivity disorder (ADHD) in children as an adjunct to conventional diagnostic methods. Psychol Res Behav Manag. 2011;4:113-117.

19. Lewis JE, Tennenbaum SL, Gao J, et al. Comparing the accuracy of ES-BC, EIS-GS, and ES Oxi on body composition, autonomic nervous system activity, and cardiac output to standardized assessments. Medical Devices: Evidence and Research. 2011;4:169-177.

20. Rand S, Petrofsky JS, Zimmerman G. Diabetes: Sweat Response and Heart Rate Variability During Electrical Stimulation in Controls and People With Diabetes. J Appl Res. 2008;8(1):48-54.

21. Vetrugno R, Liguori R, Cortelli P, Montagna P. Sympathetic skin response: basic mechanisms and clinical applications. Clin Auton Res. 2003;13(4):256-270. 
22. Sugenoya J, Iwase S, Mano T, Ogawa T. Identification of sudomotor activity in cutaneous sympathetic nerves using sweat expulsion as the effector response. Eur J Appl Physiol Occup Physiol. 1990;61(3-4): 302-308.

23. Galeffi F, Sah R, Pond BB, George A, Schwartz-Bloom RD. Changes in intracellular chloride after oxygen-glucose deprivation of the adult hippocampal slice: effect of diazepam. J Neurosci. 2004;24(18):4478-4488.

24. Kroppenstedt SN, Stover JF, Unterberg AW. Effects of dopamine on posttraumatic cerebral blood flow, brain edema, and cerebrospinal fluid glutamate and hypoxanthine concentrations. Crit Care Med. 2000;28(12):3792-3798.
25. Azmitia EC. Serotonin and brain: evolution, neuroplasticity, and homeostasis. Int Rev Neurobiol. 2007;77:31-56.

26. Mamalakis G, Kafatos A, Kalogeropoulos N, Andrikopoulos N, Daskalopulos G, Kranidis A. Prostate cancer vs hyperplasia: relationships with prostatic and adipose tissue fatty acid composition. Prostaglandins Leukot Essent Fatty Acids. 2002;66(5-6):467-477.

27. Schlereth T, Brosda N, Birklein F. Somatotopic arrangement of sudomotor axon reflex sweating in humans. Autonom Neurosci. 2005;123(1-2):76-81.

28. Girach A, Manner D, Porta M. Diabetic microvascular complications: can patients at risk be identified? A review. Int J Clin Pract. 2006;60(11):1471-1483.

\section{Publish your work in this journal}

Medical Devices: Evidence and Research is an international, peerreviewed, open access journal that focuses on the evidence, technology, research, and expert opinion supporting the use and application of medical devices in the diagnosis, treatment and management of clinical conditions and physiological processes. The identification of novel devices and optimal use of existing devices which will lead to improved clinical outcomes and more effective patient management and safety is a key feature. The manuscript management system is completely online and includes a quick and fair peer-review system. Visit http://www. dovepress.com/testimonials.php to read real quotes from authors.

Submit your manuscript here: http://www.dovepress.com/medical-devices-evidence-and-research-journal 